\title{
SOME FUNCTION SPACES OF CW TYPE
}

\author{
PETER J. KAHN ${ }^{1}$
}

\begin{abstract}
J. Milnor's result on the CW type of certain function spaces map $(X, Y)$ is extended to allow the case in which $X$ has a finite $k$-skeleton and $\pi_{i} Y=0, i>k$. One conclusion is that the self-equivalence monoid of any Postnikov stage of a finite complex has CW type. Another is that the monoid of pointed self-equivalences of a $K(\pi, 1)$ manifold has contractıb's components when $\pi$ is finitely-generated.
\end{abstract}

1. Introduction and statement of results. We shall work entirely in the category of compactly-generated Hausdorff spaces ( $k$-spaces), as described in [14], unless explicit exception is noted. Thus, for example, the set $\operatorname{map}(X, Y)$ of all maps $X \rightarrow Y$ will be topologized by first endowing it with the usual compact-open toplogy and then replacing this with the corresponding $k$-topology. For another example, a (not necessarily surjective) map of $k$-spaces will be called a fibration if it satisfies the homotopy-lifting property for all $k$-spaces.

Let $\underline{W}$ denote the class of spaces having the homotopy type of a CW complex (see [7] for basic facts about $\mathrm{CW}$ complexes). The purpose of this note is to prove the following:

1.1. ThEOREM. Let $X$ and $Y$ be connected spaces in $\underline{W}$ and $n$ a nonnegative integer such that:

(a) $X$ is homotopy-equivalent to a $C W$ complex with finite $n$-skeleton, and

(b) $\pi_{i} Y=0$, for $i>n$.

Then $\operatorname{map}(X, Y)$ belongs to $\underline{W}$.

We say that a space satisfying condition 1.1(a) has $n$-finite type. Path-connected spaces satisfying 1.1(b) will be called $(n+1)$-co-connected. In [16, 17], C. T. C. Wall gives an algebraic characterization of spaces in $\underline{W}$ that have $n$-finite type. Examples of spaces in $\underline{W}$ having $n$-finite type, for all $n$, include: finite complexes, finitelydominated complexes, nilpotent spaces with finitely-generated homology groups, and connected spaces with finite fundamental group and finitely-generated higher homotopy groups.

Received by the editors December 16, 1982.

1980 Mathematics Subject Classification. Primary 55P99.

Key' words and phrases. Homotopy type, CW complex, function space.

1 Partly supported by NSF Grant MCS-8201029.

(C)1984 American Mathematical Society $0002-9939 / 84 \$ 1.00+\$ .25$ per page 
Theorem 1.1 will be derived as an easy consequence of the following

1.2. TheOrem. Suppose that $f: X_{0} \rightarrow X_{1}$ is an n-connected map and that $Y$ is $(n+1)$-co-connected, where $X_{0}, X_{1}$, and $Y$ are connected spaces in $\underline{W}$. Then, each homotopy-fibre of the induced map $f^{*}: \operatorname{map}\left(X_{1}, Y\right) \rightarrow \operatorname{map}\left(X_{0}, Y\right)$ is either empty or contractible. If $Y$ is n-co-connected, then no homotopy-fibre is empty.

The $n$-connectivity assumption on $f$ means that $f_{*}: \pi_{i} X_{0} \rightarrow \pi_{i} X_{1}$ is injective for $i<n$ and surjective for $i \leqslant n$. The homotopy-fibre $\Phi(h ; z)$ of a (not necessarily surjective) map $h: Z_{0} \rightarrow Z_{1}$, with $z \in Z_{1}$, is the fibre over $z$ in the fibration canonically associated with $h$. For the reader's convenience, we give the explicit definition of $\Phi(h ; z)$, together with related facts, in $\S 2$.

1.3. REMARK. For spaces $X, Y$ in $\underline{W}$ with nondegenerate basepoints (see 2.5), the function space $\operatorname{map}_{*}(X, Y)$ of basepoint-preserving maps $X \rightarrow Y$ is frequently used, rather than $\operatorname{map}(X, Y)$. Both Theorems 1.1 and 1.2 apply, as stated, to $\operatorname{map}_{*}$ in place of map. The proofs are virtually the same as the ones we shall give for the unpointed case. Alternatively, one can easily deduce the pointed results from the unpointed ones by making use of the "evaluation-fibration" $\operatorname{map}(X, Y) \rightarrow Y$, in which $\operatorname{map}_{*}(X, Y)$ is the fibre over the basepoint of $Y$ (cf. 2.5).

Theorem 1.1 extends a classical result of J. Milnor [9], which implies that map $(X, Y)$ belongs to $\underline{W}$ whenever $Y$ belongs to $\underline{W}$ and $X$ has the homotopy type of a finite complex. Although this result is extremely useful, the finiteness condition on $X$ is troublesome. On the one hand, it cannot be avoided entirely [9, p. 273]. On the other hand, the condition is frequently violated by objects arising naturally in homotopy theory: e.g., Eilenberg-Mac Lane spaces $K(\pi, n)$, stages of a Postnikov tower, etc. Theorem 1.1 was motivated by such examples and can be applied to them in some cases.

To begin with, choose some $Y$ in $\underline{W}$ of type $K(G, m), m \geqslant 1$, and observe that $Y$ is $(m+1)$-co-connected.

1.4. Corollary. Suppose that $X$ is a connected space in $\underline{W}$ of $m$-finite type. Then (a) $\operatorname{map}(X, Y)$ belongs to $\underline{W}$, and (b) if $G$ is abelian, then $\operatorname{map}(X, Y)$ is homotopy equivalent to a product $\prod_{i=0}^{m} Y_{i}$, in which $Y_{i}$ is a space in $\underline{W}$ of type $K\left(H^{m-i}(X ; G), i\right)$. For example, when $m=1, \operatorname{map}(X, Y) \simeq H^{1}(X ; G) \times Y$.

Assertion 1.4(a) is an immediate consequence of Theorem 1.1. For assertion 1.4(b), a computation of Thom [15, p. 31], shows that $\operatorname{map}(X, Y)$ has the stated singular homotopy type, and 1.4(a) implies that this is the same as its homotopy type.

As a special case, take $X$ to be any $K(\pi, n)$ space in $\underline{W}$ satisfying one of the following conditions.

1.5. (1) $m<n$;

(2) $\pi$ finitely-generated, abelian;

(3) $\pi$ finitely-generated and $m=n=1$;

(4) $\pi$ finitely-presented and of type $F P_{m}$ (cf. [1]), and $m>n=1$.

Then $X$ has $m$-finite type, and Corollary 1.4 may be applied to it. 
Corollary 1.4 has a pointed analogue, which we do not state in general but instead specialize to a case that may have some independent geometric interest.

1.6. Corollary. Let $M$ be a (finite-dimensional, topological) manifold of type $K(\pi, 1)$ with $\pi=\pi_{1} M$ finitely-generated, and let $G_{*}(M)$ denote the monoid of basepoint-preserving self-equivalences of $M$. Then each component of $G_{*}(M)$ is contractible.

Note that $M$ belongs to $\underline{W}$ and has nondegenerate basepoints. Thus, since $1.5(3)$ is satisfied, we may apply the pointed version of 1.4 (a) (or of 1.1) to conclude that $G_{*}(M)$ is in $\underline{W}$. It is straightforward to compute that each component of $G_{*}(M)$ has trivial homotopy groups, and so Whitehead's Theorem then gives contractibility.

1.7. Remarks. (a) The compact-open topology on $G_{*}(M)$ is metrizable, hence already compactly-generated.

(b) When $M$ has the homotopy type of a compact manifold, Corollary 1.6 is known, proved simply by using Milnor's theorem in the above argument. However, see the next remark.

(c) There are numerous interesting examples of $K(\pi, 1)$ manifolds. For example, these arise naturally as Riemannian manifolds of constant negative curvature, or as classical knot complements. In fact, for every countable group $\pi$ of finite cohomological dimension, there is a corresponding $K(\pi, 1)$ manifold [18, p. 320]. Many of these examples do not have the homotopy type of a finite complex, even when $\pi$ is finitely-presented.

We now apply Theorem 1.1 to Postnikov stages. Note that if a space $X$ in $\underline{W}$ has $n$-finite type, then so does $X_{(n)}$, its $n$th Postnikov stage. Thus, we have

1.8. Corollary. Suppose that $X$ is a connected space in $\underline{W}$ having $n$-finite type. Then, both $\operatorname{map}\left(X_{(i)}, X_{(i)}\right)$ and $G\left(X_{(i)}\right)$, the monoid of (unpointed) self-equivalences of $X_{(i)}$, belong to $\underline{W}$ for all $i \leqslant n$.

For our final application, let $X$ be a connected space in $\underline{W}$ of $n$-finite type, and let $p_{i}: X_{(i)} \rightarrow X_{(i-1)}$ denote the $i$ th map in a Postnikov tower for $X, i \leqslant n$. Then $p_{i}$ induces a diagram:

$$
\operatorname{map}\left(X_{(i)}, X_{(i)}\right) \overbrace{\lessgtr} \stackrel{\left(p_{i}\right)^{*}}{\operatorname{map}\left(X_{(i)}, X_{(i-1)}\right)} \underbrace{\left(p_{i}\right)^{*}}_{\left(X_{(i)}\right)-\rightarrow G\left(X_{(i-1)}\right) \subseteq} \operatorname{map}\left(X_{(i-1)}, X_{(i-1)}\right)
$$

1.10. Corollary. (a) There exists a map $\alpha: G\left(X_{(i)}\right) \rightarrow G\left(X_{(i-1)}\right)$ completing (1.9) (i.e., making it commute up to homotopy).

(b) Such an $\alpha$ is unique, up to homotopy.

(c) $\alpha$ is an $A_{\infty}$-map of monoids (in the sense of [13]).

Theorem 1.1 implies that each of the spaces of (1.9) belongs to $\underline{W}$. Theorem 1.2 then implies that $\left(p_{i}\right)^{*}$ is a homotopy equivalence. This immediately gives $1.10(\mathrm{a})$, (b). The proof of (c) is given in $\S 4$. 
1.11. Remarks. In the category of simplicial sets, there is a canonical and functorial Postnikov-tower-construction, due to J. Moore (e.g., see [8]), which yields almost immediately the existence of a simplicial analogue of $\alpha$. This analogue is a homomorphism of simplicial monoids.

In the topological category, the Postnikov-tower-construction is neither canonical nor functorial, so the existence of $\alpha$ cannot be established so readily.

If we apply the topological realization functor to the simplicial analogue mentioned above, we do obtain a suitable $\alpha$, provided that we know that $G\left(X_{(i)}\right)$ has $C W$ type, which requires Theorem 1.1. Thus, this gives an alternative proof of 1.10(a). A third proof will arise (also depending on 1.1) in the process of demonstrating 1.10 (c) in $\S 4$.

Note that $1.10(\mathrm{c})$ is as close as one can reasonably expect to get to asserting that $\alpha$ is a homomorphism of monoids.

2. Some technical facts. This section presents the technical information needed for the proofs of the theorerns and of Corollary $1.10(\mathrm{c})$.

2.1. Definitions. Given a map $h: Z_{0} \rightarrow Z_{1}$, set

$$
E_{h}=\left\{(z, \omega) \in Z_{0} \times \operatorname{map}\left(I, Z_{1}\right) \mid \omega(0)=h(z)\right\},
$$

and define $p_{h}: E_{h} \rightarrow Z_{1}$ by $p_{h}(z, \omega)=\omega(1)$. Here $I$ is the unit interval $[0,1]$. $Z_{0} \times \operatorname{map}\left(I, Z_{1}\right)$ and $E_{h}$ are topologized in accordance with the usual conventions concerning the $k$-topology (cf. [14]). In particular, the $k$-topology on $E_{h}$ coincides with the relative topology induced by the $k$-space $Z_{0} \times \operatorname{map}\left(L, Z_{1}\right)$.

In the classical context (i.e., in which the compact-open topology is used, etc.), $p_{h}$ is a Hurewicz fibration (e.g., cf. [11, pp. 99-100]). It follows easily that if we then impose $k$-topologies, $p_{h}$ becomes a fibration in the sense we describe in the introduction. Note that image $p_{h}$ is the union of all path-components of $Z_{1}$ meeting image $h$.

The homotopy-fibre $\Phi\left(h ; z_{1}\right)$ is defined to be $p_{h}^{-1}\left(z_{1}\right)$, for each $z_{1} \in Z_{1}$.

We use " $\simeq$ " to denote homotopy-equivalence.

2.2. Lemma. If $p: E \rightarrow B$ is a fibration and $b \in B$, then $p^{-1}(b) \simeq \Phi(p ; b)$.

2.3. Lemma. Suppose that

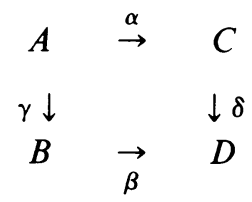

is a homotopy-commutative diagram in which $\alpha$ and $\beta$ are homotopy-equivalences. Then, for every $x \in B, \Phi(\gamma ; x) \simeq \Phi(\delta ; \beta(x))$.

Both lemmas are folklore in homotopy theory. The Eckmann-Hilton dual of a special case of 2.3 is proved in [6], and another special case follows from results in [10]. 
2.4. Lemma. Suppose that $p: E \rightarrow B$ is a fibration and $B \in \underline{W}$. Then $E \in \underline{W}$ if and only if each $p^{-1}(b) \in \underline{W}$.

Except for our use of the $k$-topology, this lemma follows immediately from results of Stasheff [12] (more precisely, from his Propositions (0) and (12), together with our Lemma 2.2). Stasheff's arguments remain correct in our $k$-topology-context; in fact, they may be somewhat abbreviated here.

Note that these lemmas do not require maps to be surjective.

2.5. Definitions. (a) Suppose that $f: A \rightarrow X$ is a closed cofibration in $\underline{W}$, and consider the induced map $f^{*}: \operatorname{map}(X, Y) \rightarrow \operatorname{map}(A, Y)$, for any $Y$ in $W$. In the classical case (i.e., compact-open topology), this is a Hurewicz fibration [11, p. 97]. After imposing the $k$-topology, it becomes a fibration in our sense. We denote the fibre over $g \in \operatorname{map}(A, Y)$ by $\operatorname{map}(X, Y ; g)$. When $f$ is an inclusion map, we may think of $\operatorname{map}(X, Y ; g)$ as consisting of all extensions $\bar{g}: X \rightarrow Y$ of $g$.

Note that if $A$ is a point, so that, by definition, $f$ maps onto a nondegenerate basepoint $*$, then $f^{*}$ may be identified with the evaluation $\operatorname{map} \operatorname{map}(X, Y) \rightarrow Y$, which sends $\bar{g}$ to $\bar{g}(*)$.

(b) Using the above notation, suppose that $(X, A)$ is a CW pair, $A \neq \varnothing$, and $f$ is the inclusion map (henceforth suppressed). As above, choose some $g: A \rightarrow Y$ and some extension $\bar{g} \in \operatorname{map}(X, Y ; g)$. Finally, pick basepoints $x \in A \subseteq X$ and $y=$ $g(x) \in Y$. Then, $\bar{g}$ determines the structure of a $\mathbf{Z} \pi_{1}(X, x)$-module on $\pi_{m}(Y, y)$, for any $m \geqslant 2$, which we denote by $\bar{g}^{\#} \pi_{m} Y$.

In Lemma 2.6, $i$ is any fixed nonnegative integer, and cohomology will be taken with local coefficients.

2.6. Lemma. Assume the context of $2.5(\mathrm{~b})$, and, in addition, suppose (a) the inclusion $A \hookrightarrow X$ is 1-connected, and (b) $H^{n}\left(X, A ; \bar{g}^{\#} \pi_{n+i} Y\right)=0$, for all $n+i \geqslant 2$. Then, $\pi_{i}(\operatorname{map}(X, Y ; g))=0$, where the homotopy group $\pi_{i}$ is based at $\bar{g}$ when $i \geqslant 1$.

Proof. A typical element of $\pi_{i}(\operatorname{map}(X, Y ; g))$ is represented by a map $\phi$ : $S^{i} \rightarrow \operatorname{map}(X, Y ; g)$, based at $\bar{g}$, which we may transform, by adjointness to a map $\tilde{\phi}$ : $S^{i} \times X \rightarrow Y$. The conclusion $\pi_{i}=0$ is then seen to be equivalent to the existence, for each $\phi$, of an extension $\psi$ in the diagram

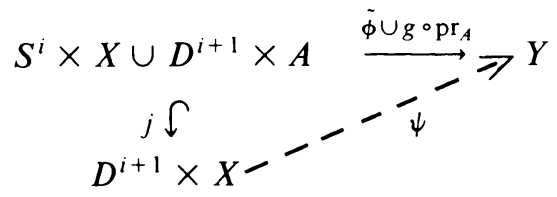

where $\operatorname{pr}_{A}: D^{i+1} \times A \rightarrow A$ is the canonical projection. The hypotheses imply that $j$ induces a $\pi_{1}$-isomorphism, so that $\psi$ may be defined on the 2-skeleton. Further obstructions to this extension lie in zero groups.

For each $i \geqslant 0$, define the relative $i$-skeleton $\bar{X}^{i}$ to be $A \cup \cup$ cells of $X$ of dimension $\leqslant i\}$. The inclusions $\bar{X}^{i} \subseteq \bar{X}^{i+1}$ induce maps $\operatorname{map}\left(\bar{X}^{i+1}, Y ; g\right) \rightarrow$ $\operatorname{map}\left(\bar{X}^{i}, Y ; g\right)$ making $\left\{\operatorname{map}\left(\bar{X}^{i}, Y ; g\right)\right\}$ into an inverse limit system. 


\subsection{Lemma. Restriction induces a homeomorphism}

$$
\operatorname{map}(X, Y ; g) \approx{\underset{\lim }{i}}_{i} \operatorname{map}\left(\bar{X}^{i}, Y ; g\right) .
$$

The map induced by restriction is easily seen to be continuous and bijective, and so the burden of proof is to check that it is open. We leave this to the reader.

2.8. LEMMA. If, in the tower

$$
* \stackrel{p_{1}}{\leftarrow} E_{1} \stackrel{p_{2}}{\leftarrow} E_{2} \stackrel{p_{3}}{\leftarrow} E_{3} \leftarrow \cdots
$$

of fibrations, each $E_{i} \simeq *$, then $\lim E_{i} \simeq *$.

Proof. Because each $E_{i} \simeq *$, each $p_{i}$ is surjective, so that $\lim _{\leftarrow} E_{i} \neq \varnothing$. Choose $\left\{e_{i}\right\} \in \lim E_{i}$, and set $F_{i}=p_{i}^{-1}\left(e_{i-1}\right) \subseteq E_{i}$. Because the fibrations are fibre-homotopy trivial, each $F_{i} \simeq *$.

We define a family of contractions $h_{i}: E_{i} \times[0,1] \rightarrow E_{i}, i \geqslant 1$, satisfying (i) $h_{i}(x, t)=e_{i}$, for all $(x, t) \in E_{i} \times\left[0,2^{-i}\right]$, and (ii) $p_{i} h_{i}=h_{i-1}\left(p_{i} \times \mathrm{id}_{[0,1]}\right)$. By the universal property for $\lim _{\leftarrow}$ (in the category of $k$-spaces), the $h_{i}$ fit together to give a contraction of $\underset{\leftarrow}{\lim } E_{i}$. More precisely, $\lim h_{i}$ is a continuous map $\underset{\leftarrow}{\lim }\left(E_{i} \times[0,1]\right)$ $\rightarrow \lim E_{i}$, which, when composed with the natural homeomorphism

$$
\left(\lim _{\leftarrow} E_{i}\right) \times[0,1] \rightarrow \lim \left(E_{i} \times[0,1]\right),
$$

yields the desired contraction. It remains to define the $h_{i}$.

Define $h_{1}$ to be any contraction satisfying (i) for $i=1$, and suppose $h_{j-1}$ is defined satisfying (i), (ii) for $i=j-1$. Use homotopy-lifting to obtain a map $h_{j}^{\prime}$ : $E_{j} \times[0,1] \rightarrow E_{j}$ such that $h_{j}^{\prime}$ satisfies (ii) for $i=j$, and $h_{j}^{\prime}(x, 1)=x$, for all $x \in E_{j}$. Clearly, $h_{j}^{\prime}\left(E_{j} \times\left[0,2^{-j+1}\right]\right) \subseteq F_{j}$. Use the contractibility of $F_{j}$ to define a deformation $d_{t}$ in $F_{j}$ between the trivial map $E_{j} \rightarrow\left\{e_{j}\right\}$ and $h_{j}^{\prime} \mid E_{j} \times\left\{2^{-j+1}\right\}$. Then, define $h_{j}$ as follows:

$$
h_{j}(x, t)= \begin{cases}e_{j}, & 0 \leqslant t \leqslant 2^{-j} \\ d_{s}(x), & 2^{-j} \leqslant t \leqslant 2^{-j+1}, s=2^{j}\left(t-2^{-j}\right), \\ h_{j}^{\prime}(x, t), & 2^{-j+1} \leqslant t \leqslant 1 .\end{cases}
$$

Clearly, $h_{j}$ is a contraction satisfying (i), (ii) for $i=j$.

This completes the technical information needed for our proofs of Theorems 1.1 and 1.2. The remaining facts will be used for our proof of Corollary 1.10(c).

We shall make use of the Moore loop space $\Lambda Z$ of a space $Z$, which has the same homotopy type as the ordinary loop space and has a strictly associative loop composition (cf. [13, p. 14]).

Consider the self-equivalence monoid $G(X)$ of a space $X$ in $\underline{W}$, and let $B G(X)$ denote the classifying space constructed by Dold and Lashof [3].

2.9. FACT. If $G(X)$ is in $W$, then there is an $A_{\infty}$-equivalence of monoids $G(X) \approx \Lambda B G(X)$. 
The Dold-Lashof construction produces a weak homotopy equivalence $G(X) \rightarrow$ $\Lambda B G(X)$ (see [13, Theorem 4.3]). Since $G(X)$ is in $\underline{W}$, an argument of Stasheff [12, p. 243], implies that $B G(X)$ is in $\underline{W}$, and then Milnor's theorem yields the same for $\Lambda B G(X)$. It follows that the weak equivalence is a homotopy equivalence. Work of Stasheff and of Fuchs (cf. [13, pp. 33-35]) then shows that this homotopy equivalence is an $A_{x}$-equivalence of monoids.

2.10. FaCt. Suppose that $X$ and $G(X)$ are in $\underline{W}$, as above. Then $B G(X)$ classifies $X$-fibrations over spaces in $\underline{W}$.

Stasheff [12] proves such a result, without using $k$-topologies, under the assumption that $X$ is a finite complex. The finiteness assumption insures that certain maps in Stasheff's constructions are continuous and that $G(X)$ belongs to $\underline{W}$, and this is

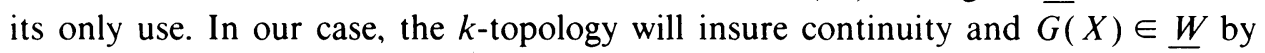
hypothesis. Thus, Stasheff's argument yields 2.10 .

An alternative proof follows from Dold's representability theorem [2], obtaining a space $B(X)$ in $\underline{W}$ which classifies $X$-fibrations. The assumption $G(X) \in \underline{W}$ is then needed to verify that $B(X) \simeq B G(X)$.

\section{Proofs of the theorems.}

3.1. Theorem $1.2 \Rightarrow$ Theorem 1.1. We may take $X$ to be a CW complex with finite $n$-skeleton, $X^{n}$, and we let $f: X^{n} \rightarrow X$ be the inclusion. Then $f^{*}: \operatorname{map}(X, Y) \rightarrow$ $\operatorname{map}\left(X^{n}, Y\right)$ is a fibration. By 1.2, together with 2.2, the fibres of $f^{*}$ are either empty or contractible: in either case they belong to $\underline{W}$. Since $\operatorname{map}\left(X^{n}, Y\right) \in \underline{W}$, by Milnor's theorem, Lemma 2.4 now gives the desired conclusion.

3.2. Proof of Theorem 1.2. The proof proceeds in a number of steps.

3.2.1. We observe first that we may assume that $\left(X_{1}, X_{0}\right)$ is a CW pair with $X_{0}$ containing the $n$-skeleton of $X_{1}$ and $f$ the inclusion $X_{0} \rightarrow X_{1}$. For in the general case, one can always find such a $\mathrm{CW}$ pair $\left(K_{1}, K_{0}\right)$ and a homotopy-commutative square

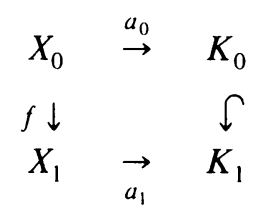

in which each $a_{i}$ is a homotopy-equivalence. Apply map $(-, Y)$ to this square, and then apply Lemma 2.3 to the result.

Henceforth, we assume that the above reduction has been made. Moreover, in light of Lemma 2.2, it suffices to verify the conclusions of Theorem 1.2 for each honest fibre of the fibration $f^{*}: \operatorname{map}\left(X_{1}, Y\right) \rightarrow \operatorname{map}\left(X_{0}, Y\right)$. Finally, we may suppose that $n>0$, because when $n=0, Y$ is contractible, and the result is trivially true.

3.2.2. Case 1. $X_{1}=X_{0} \cup_{\phi} D^{p}, p>n$. Choose $a \in \operatorname{map}\left(X_{0}, Y\right)$. When $Y$ is $n$-coconnected, $a$ extends over $X_{1}$, so that $\left(f^{*}\right)^{-1}(a)=\operatorname{map}\left(X_{1}, Y ; a\right)$ is then nonempty. In any case, $\operatorname{map}\left(X_{1}, Y ; a\right)$ is homeomorphic to $\operatorname{map}\left(D^{p}, Y ; a \phi\right)$, which we henceforth denote by $M$, and which is the fibre over $a \phi$ in the restriction-fibration $\operatorname{map}\left(D^{p}, Y\right) \rightarrow \operatorname{map}\left(S^{p-1}, Y\right)$. By Milnor's theorem and Lemma 2.4, this fibre $M$ 
belongs to $\underline{W}$, so that, if it is nonempty, we need only show that $\pi_{i} M=0$, for all $i \geqslant 0$. But this follows from Lemma 2.6, together with the $(n+1)$-co-connectivity of $Y$, which completes the proof in this case.

3.2.3. Case 2. $X_{1}=X_{0} \cup_{\phi} \sqcup_{\alpha} D_{\alpha}^{p}, p>n$. Set $\phi_{\alpha}=\phi \mid S_{\alpha}^{p-1}$. Then, $\left(f^{*}\right)^{-1}(a)$ is homeomorphic to $\Pi_{\alpha} \operatorname{map}\left(D_{\alpha}^{p}, Y ; a \phi_{\alpha}\right)$ (cf. Lemma 2.7). Case 1 shows that each factor in this product is empty or contractible. Hence, so is $\left(f^{*}\right)^{-1}(a)$.

3.2.4. Case 3. $\left(X_{1}, X_{0}\right)$ is a CW pair with cells in $X_{1} \backslash X_{0}$ having bounded dimension $>n$. We have a filtration of $X_{1}$ by relative skeleta (cf. §2)

$$
X_{0}=\bar{X}^{n} \subset \bar{X}^{n+1} \subset \cdots \subset \bar{X}^{q}=X_{1},
$$

for some $q \leqslant n, q<\infty$. Apply map $(-, Y)$ to this filtration, obtaining a finite tower of fibrations in which each fibre is empty or contractible, either because of 3.2 .3 or because it is a point. The total fibre over $a,\left(f^{*}\right)^{-1}(a)$, if nonempty, is itself then fibred

$$
*=\operatorname{map}\left(\bar{X}^{n}, Y ; a\right) \leftarrow \operatorname{map}\left(\bar{X}^{n}+1, Y ; a\right) \leftarrow \cdots \leftarrow \operatorname{map}\left(\bar{X}^{q}, Y ; a\right)=\left(f^{*}\right)^{-1}(a)
$$

by a tower of fibrations whose fibres are selected from the (nonempty) fibres above. Hence, they are contractible, and an easy induction shows that $\left(f^{*}\right)^{-1}(a)$ is contractible.

3.2.5. Case 4. $\left(X_{1}, X_{0}\right)$ is a CW pair such that $X_{0}$ contains the $n$-skeleton of $X_{1}$. When $\left(f^{*}\right)^{-1}(a)$ is nonempty, use 3.2 .4 to obtain a (possibly infinite) tower of fibrations

$$
*=\operatorname{map}\left(\overline{X^{n}}, Y ; a\right) \leftarrow \operatorname{map}\left(\bar{X}^{n+1}, Y ; a\right) \leftarrow \cdots
$$

in which each total space is contractible. The desired result now follows immediately from Lemmas 2.7 and 2.8 .

In light of 3.2.1, the proof of Theorem 1.2 is now complete.

4. Proof of Corollary 1.10(c). Recall that $X$ is a connected space in $\underline{W}$ of $n$-finite type and $X_{(j)}$ denotes its $j$ th Postnikov stage. When $j \leqslant n, G\left(X_{(j)}\right)$ belongs to $\underline{W}$ (Corollary 1.8), so that we may make use of Facts 2.9 and 2.10. To begin with, we shall identify $G\left(X_{(j)}\right)$ with $\Lambda B G\left(X_{(j)}\right)$ via the $A_{\infty}$-equivalence of 2.9 .

Now let $i \leqslant n$ be as in the statement of 1.10 . The proof proceeds by defining a map

$$
B G\left(X_{(i)}\right) \stackrel{\beta}{\rightarrow} B G\left(X_{(i-1)}\right)
$$

such that $\Lambda \beta$ completes (1.9) (thus giving a third proof of Corollary 1.10(a)). $\Lambda \beta$ is, of course, an $A_{\infty}$-map of monoids.

Any map $\alpha: G\left(X_{(i)}\right) \rightarrow G\left(X_{(i-1)}\right)$ completing (1.9) is homotopic to $\Lambda \beta$, by Corollary $1.10(\mathrm{~b})$, and this finishes the proof, because $A_{\infty}$-maps of monoids are closed under homotopy $[13$, p. 33].

It remains to define $\beta$ with the requisite property. By 2.10 , there is a universal $X_{(i)}$-fibration $E G\left(X_{(i)}\right) \rightarrow B G\left(X_{(i)}\right)$. Its $(i-1)$ st Moore-Postnikov stage is an $X_{(i-1)}$-fibration over $B G\left(X_{(i)}\right)$, which may be classified by a map $B G\left(X_{(i)}\right) \rightarrow$ $B G\left(X_{(i-1)}\right)$ because $B G\left(X_{(i)}\right)$ is in $\underline{W}$. This classifying map is $\beta$. 
The argument that $\Lambda \beta$ completes (1.9) has two steps, whose details we leave to the reader: (1) if $f: B \rightarrow B G\left(X_{(i)}\right)$ classifies the $X_{(i)}$-fibration $p: E \rightarrow B$, verify that $\beta \circ f$ classifies the $(i-1)$ st Moore-Postnikov stage of $p$. (2) When $B$ is a suspension $\Sigma A$, the fibration $p: E \rightarrow B$ is classified by a "clutching function" $g: A \rightarrow G\left(X_{(i)}\right)$. Using (1), together with standard arguments, verify that the $(i-1)$ st Moore-Postnikov stage of $p$ is then classified by $\Lambda \beta \circ g$. This readily implies that $\Lambda \beta$ completes (1.9) and concludes our proof.

\section{REFERENCES}

1. K. Brown, Cohomology of groups, Graduate Texts in Math., no. 87, Springer-Verlag, Berlin and New York, 1982.

2. A. Dold, Halbexacte homotopiefunktoren, Lecture Notes in Math., vol. 12, Springer-Verlag, Berlin and New York, 1966

3. A. Dold and R. Lashof, Principal quasifibrations and fibre homotopy equivalence of bundles, Illinois J. Math. 3 (1959), 285-305.

4. Martin Fuchs, A modified Dold-Lashof construction that does classify H-principal fibrations, Math. Ann. 192 (1971), 328-340.

5. The functor [ , Y] and loop fibrations, I, Michigan Math. J. 14 (1967), 283-287.

6. P. J. Hilton, Homotopy theory and duality, Gordon \& Breach, New York, 1966.

7. A.Lundell and S. Weingram, The topology of $C W$ complexes, Van Nostrand, Princeton, N. J., 1969.

8. J. P. May, Simplicial objects in algebraic topology, Van Nostrand Mathematical Studies, no. 11, Van Nostrand, Princeton, N. J., 1967.

9. J. W. Milnor, On spaces having the homotopy type of a CW complex, Trans. Amer. Math. Soc. 90 (1959), 272-280.

10. Y. Nomura, On extensions of triads, Nagoya Math. J. 27 (1966), 249-277.

11. E. Spanier, Algebraic topology, McGraw-Hill, New York, 1966.

12. J. Stasheff, A classification theorem for fibre spaces, Topology 2 (1963), 239-246.

13. , $H$-spaces from a homotopy point of view, Lecture Notes in Math., vol. 161, Springer-Verlag, Berlin and New York, 1970.

14. N. Steenrod, A convenient category of topological spaces, Michigan Math. J. 14 (1967), 133-152.

15. R. Thom, L'homologie des espaces fonctionnels, Colloque de Topologie Algẹbrique, Louvain, 1956,

G. Thone, Liège, 1957, pp. 29-39.

16. C. T. C. Wall, Finiteness conditions on CW complexes. I, Ann. Math. 81 (1965), 56-69.

17. 129-139.

18. Group actions on manifolds, Topology of Manifolds (J. Cantrell and C. Edwards, eds.), Markham, Chicago, Ill., 1970, pp. 319-333.

Department of Mathematics, Cornell University, IthaCa, New York 14853 